\title{
Galactic Bulge VHE Tau-neutrino and Gamma-ray Monitor with Ashra-1 and NTA detectors
}

\author{
Satoru Ogawa ${ }^{1, *}$ and Makoto Sasaki ${ }^{2}$ \\ ${ }^{1}$ Department of Physics, Toho University, Funabashi, Chiba 274-8510, Japan \\ ${ }^{2}$ Institute for Cosmic Ray Research, University of Tokyo, Kashiwa, Chiba 277-8582, Japan \\ E-mail: ogawa@ph.sci.toho-u.ac.jp, sasakim@icrr.u-tokyo.ac.jp
}

The Ashra phase-1 (Ashra-1) detector has been developed to efficiently capture fine detail images from air-shower (AS) Cherenkov (CE) and fluorescence (FL) light induced by the Earth-skimming $v_{\tau}$ and $\gamma$-ray ASs. Based on the performance of Ashra-1, we are planning a new extension, namely the Neutrino Telescope Array (NTA), an AS imaging $v$ and $\gamma$-ray observing system for Unambiguous Discovery and Identification of Non-thermal Hadronic Processes in the Universe. The four NTA stations are planned to be located on Mauna Loa at 3000-3500 m a.s.l. (layout of the NTA Summit Array). Using the four stations, it will monitor AS phenomena occurring in the volume of air above the surface, including the largest volcano, Mauna Loa, the Big Island of Hawaii and the surrounding ocean, and can efficiently detect CE and FL light from $v_{\tau}$ ASs with both short and long decay lengths and $\gamma$-ray ASs. The sensitivity of NTA $v_{\tau}$ with detailed MC is sufficient to probe the Pevatron as an extension of astrophysical neutrino flux detected by IceCube and a prediction of cosmogenic neutrino. The point-back accuracy is evaluated to be within $0.2^{\circ}$ of the original direction of $\mathrm{ES} v_{\tau}$ 's on the PeV scale. As a first step in the deployment of a minimal systematic detector, we propose to simultaneously observe the $10 \mathrm{TeV}-10 \mathrm{PeV} \gamma$-rays from the Galactic bulge (GB) with Ashra-1 and the Earth-skimming $v_{\tau}$ 's with NTA to clearly identify the Pevatrons and comprehensively understand the emission process there. The effective detection area of Ashra- 1 and that of NTA for the Galactic bulge $\gamma$-rays with the energies around $1 \mathrm{PeV}$ is more than 10 and 100 times respectively larger than that of a $500 \mathrm{~m}$-scale ground array. The GB can also be considered an intriguing testing site for the discovery of elementary particles predicted by the non-standard theory of particle physics. NTA can perform the sensitive indirect search for PeV-scale WIMP dark matter (DM) and non-standard new particles, with much better effective detection area for tau neutrinos from DM annihilation in the GB, especially above $10 \mathrm{PeV}$, the complementary sensitive energy region for IceCube.

$37^{\text {th }}$ International Cosmic Ray Conference (ICRC 2021)

July 12 th - 23rd, 2021

Online - Berlin, Germany

\footnotetext{
${ }^{*}$ Presenter
} 


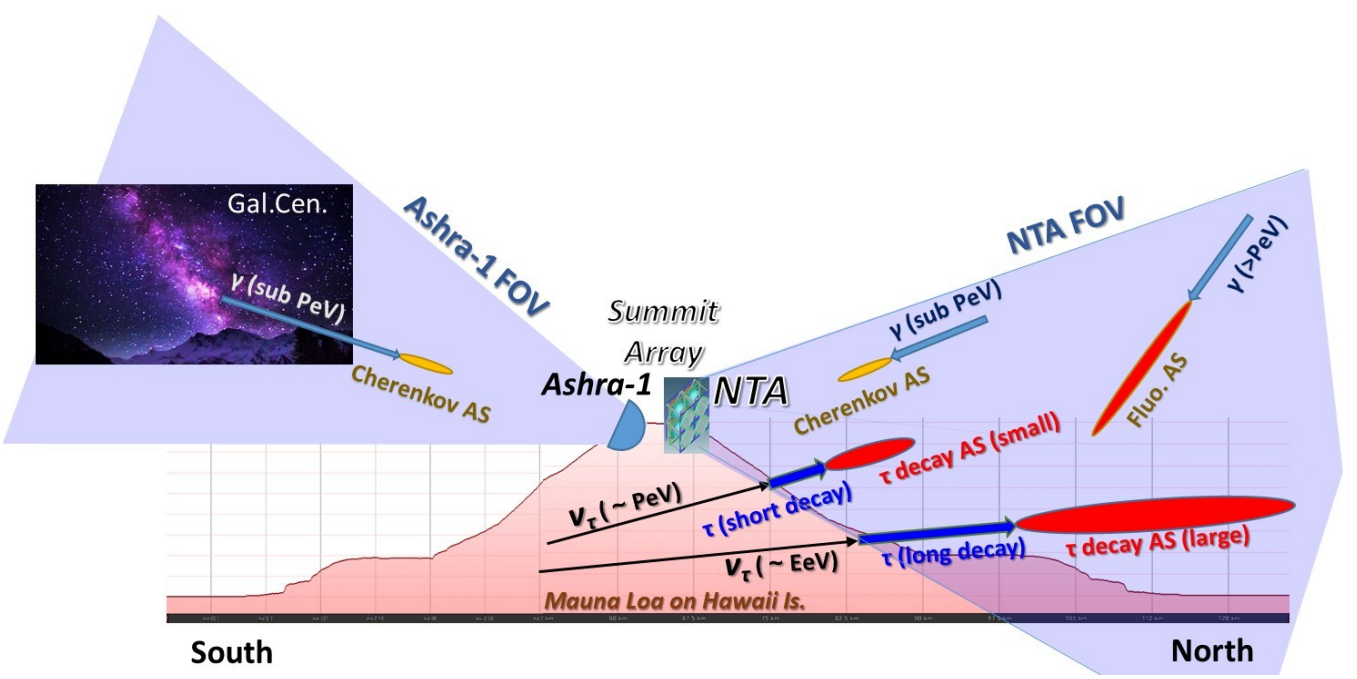

Figure 1: Schematic of imaging observations of PeV v's, $\gamma$-rays, and nuclei with Ashra NTA Summit Array. Six Ashra-1 collectors will continuously observe the sky region through which the Galactic bulge passes during the night. The NTA can confirm the coincidence of the source of ES- $\nu_{\tau}$ 's with that of $\gamma$-ray events by Ashra-1.

\section{Introduction}

The combined detection of PeV $v$ 's and $\gamma^{\prime}$ 's from the accelerator is essential to identify the location and physical mechanism $p+\gamma \rightarrow \Delta^{+} \rightarrow \pi^{0}+p, \pi^{+}+n ; p+$ nucleus $\rightarrow \pi^{ \pm, 0}+X$, which can reveal the origin of the long unsolved cosmic rays. Recently several suggestive observations have been made independently [1-3]. Such a "multi-particle" paradigm [4] can be implemented with Ashra NTA, which has a single unique detector system [5].

In 2002, Sasaki et al. announced the possibility of a distinctive Earth-skimming tau $v\left(\mathrm{ES}-v_{\tau}\right)$ technique [15]. A large target mass is obtained by detecting the air-shower (AS) produced by $\tau$ decay in the air. The $\tau$ produced by the interaction of $v_{\tau}$ with matter on the earth comes out by traversing mountains and ground. In addition to the good detection sensitivity of $v_{\tau}$, the advantages include complete shielding of the cosmic ray secondary, accurate determination of the direction of arrival, and neglect of the background from atmospheric $v$ 's [16]. The Ashra NTA detector can efficiently image AS Cherenkov (CE) light and fluorescence (FL) light generated from ES- $v_{\tau}$ and $\gamma$ ASs in an effective volume of air within the field of view (FOV) (Figure 1). A unique feature is the ability to identify $v$ and $\gamma$ point sources with a resolution higher than about $0.1^{\circ}$ and the strong background hadron rejection in $\gamma$ selection, in both FL and CE light.

\section{From Ashra-1 to Ashra NTA}

The Ashra Phase 1 (Ashra-1) [17] light collector (LC) achieves the total resolution of 3 arcminutes $\left(0.05^{\circ}\right)$ covering $42^{\circ}$ FOV. Its main technical feature is the use of an electrostatic lens rather than an optical lens to produce a converging beam in a 20-inch photoelectric lens imaging tube (PLI) [18]. The light is reduced to 1 inch size at the focal plane to achieve high resolution with 


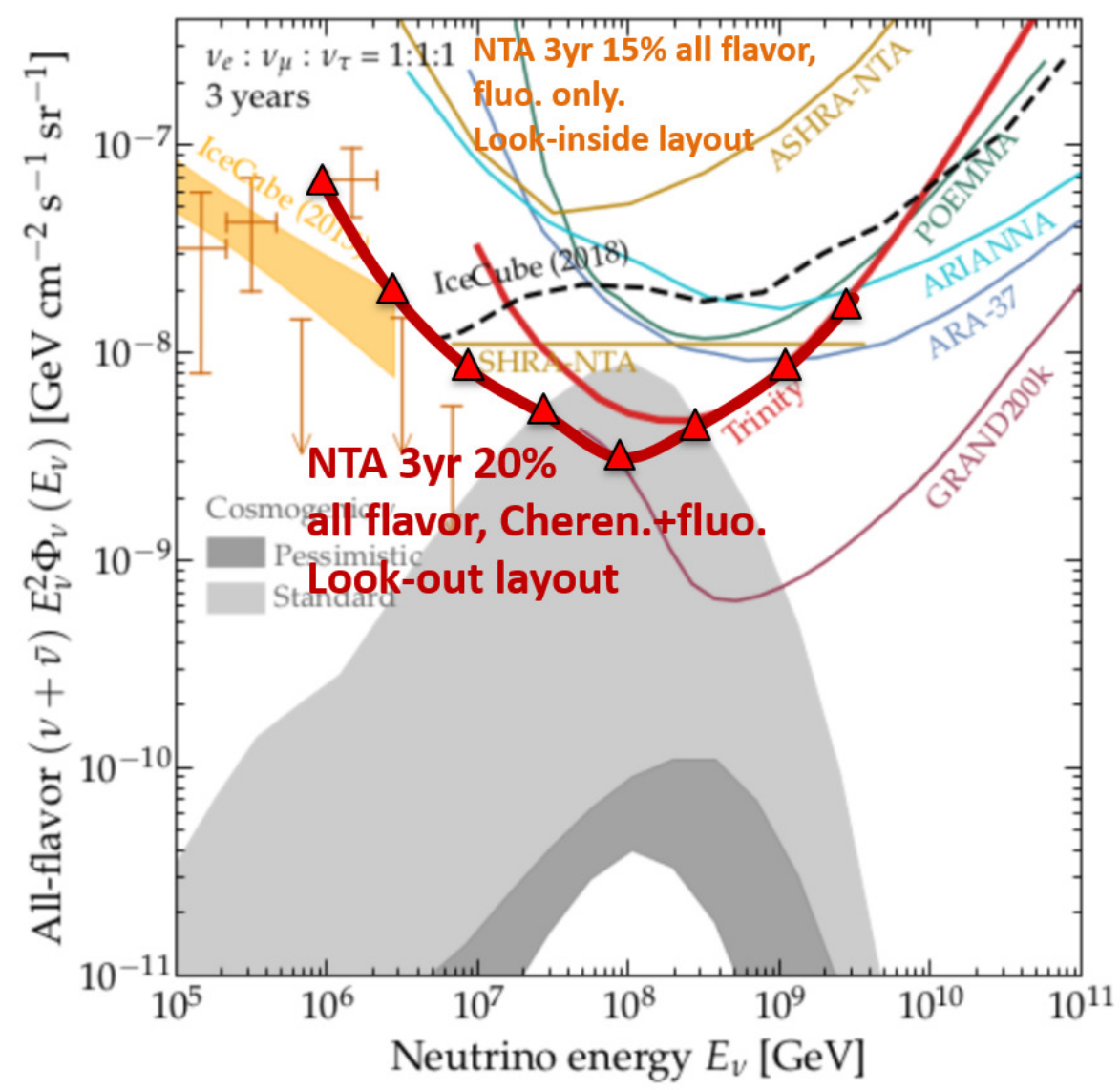

Figure 2: Sensitivity of Ashra with only fluorescence mode, that including far-Cherenkov mode and other experiments, neutrino flux predictions, and existing flux constraints. Figure adapted from [21].

a wide FOV [19]. The subsequent triggered readout photoelectric image pipeline (PIP) [20] can image and read out three independent phenomena at different time scales, i.e., AS CE emission (ns), AS FL ( $\mu$ s), and star light (s), without sacrificing the signal-to-noise ratio. Since 2008, we have been conducting a demonstration experiment at Mauna Loa Observatory, located at an altitude of 3300 meters on the Big Island of Hawaii. Following the alert of GRB081203A by the SWIFT satellite, Ashra-1 was the first to successfully search for PeV-EeV $v_{\tau}$ from GRBs using the ES- $v_{\tau}$ method and set a stringent fluence limit [22]. The updated ES- $v_{\tau}$ limits from 1863 hours of observations will be published soon [23].

Based on the performance of Ashra-1, i we have planned a new extension, the Ashra NTA, which is an AS imaging $v$ and $\gamma$ observing system aimed at the unambiguous discovery and identification of non-thermal hadronic processes in the Galactic, extragalactic, or cosmogenic Universe [5]. Based on detailed simulation studies [5, 16], the layout of the NTA stations is optimized to increase the detection sensitivity of ES- $v_{\tau}$ around $1 \mathrm{PeV}$, and four NTA stations will be set up at Mauna Loa at 3000-3500 m altitude, While monitoring the atmosphere around Mauna Loa, including the surface of Mauna Loa, the largest volcano, four NTA stations (NTA Summit Array) will be placed at Mauna Loa, 3000-3500 m above sea level, to efficiently detect CE and FL light 


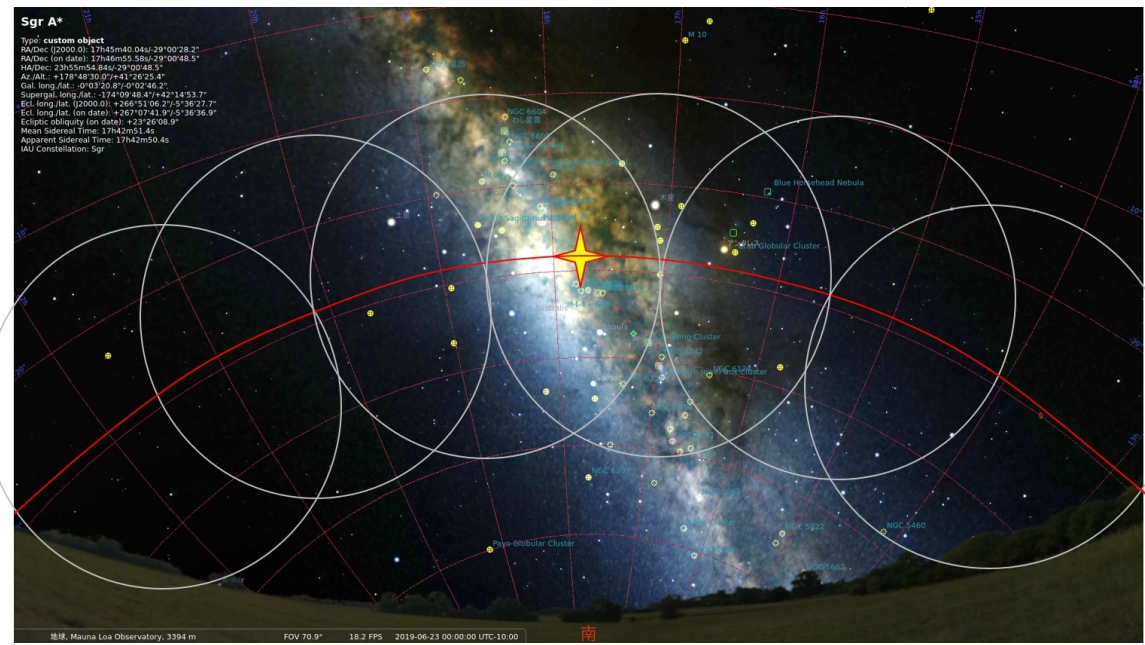

Figure 3: Simulated southern sky at the Mauna Loa site at 0:00 on June 23, 2019. The cross star indicates the location of the Galactic center(GC). The track of GC (arc) and the FOV of the rearranged Ashra-1 light collectors (circles) are also shown.

generated from $\tau$ AS with short and long decay lengths and $\gamma$ AS (Figure 1). The reconstructed AS images with fine resolution is powerful not only in the determination of point sources of $\mathrm{PeV}$ $v_{\tau}$ 's but also FL observation for $\gamma \mathrm{ASs}$ above $\mathrm{PeV}$ with the large effective area (Figure 1). Figure 2 shows a comparison of NTA's total $v_{\tau}$ detection sensitivity with that of NTA with only fluorescence mode [5] Trinity [21], GRAND [6], POEMMA [7], ARIANNA [8], and ARA-37 [9]. Also shown are predictions of the cosmogenic neutrino flux [10], measurements of the astrophysical neutrino flux with IceCube [11], and the recent limits from IceCube [12], AUGER [13], and ANITA [14]. The NTA $v_{\tau}$ sensitivity is sufficient to probe Pevatorons, an extension of the IceCube detected astrophysical neutrino flux and predictions of the cosmogenic neutrino flux. Adding that the unique combination among CE and FL observations for both ES- $\nu_{\tau}$ 's and $\gamma$ with NTA will truly identify Pevatron(s) and open up new types of search for $\nu$ 's and $\gamma$ 's in the wide energy range.

As the first step, the combination between Ashra-1 light collectors and new NTA detector units is useful to realize the comprehensive observation both with $\mathrm{TeV}-\mathrm{PeV} \gamma$-rays and $\mathrm{PeV} v$ 's. Six Ashra-1 LCs will be realigned for the FOV centers to be on the arc of the Galactic Center trajectory optimizing the monitoring coverage and the stereoscopic observation efficiency as shown in Figure 4. Therefore we will arrange the fields of view so that their fields of view overlap by half of the field of view of adjacent telescopes as shown in Figure 4. As a result, the total rate of the stereoscopic observation can be more than $70 \%$ of the fully-covered trajectory in the sky. We estimate the annual observable time to be 1150 hours $\times \epsilon_{w}$ during nights without moon in the south, where the weather efficiency $\epsilon_{w}>90 \%$ according to the Ashra-1 experience, which is more than 50 times better than HESS achieved i.e. 227 hours for Sgr A* in 10 years [2]. The series of 6 Ashra-1 LC FOVs can monitor AS CE light of $\gamma$-rays arrived from the Galactic bulge (GB) or central region. Another advantage of the detection of $\gamma$-rays with Ashra-1 is the large zenith-angle method. The GB trajectory experiences the zenith angle larger than 50 degrees (70 degrees), which corresponds to the distance to the shower max larger than $9 \mathrm{~km}(26 \mathrm{~km})$ and the threshold energies 


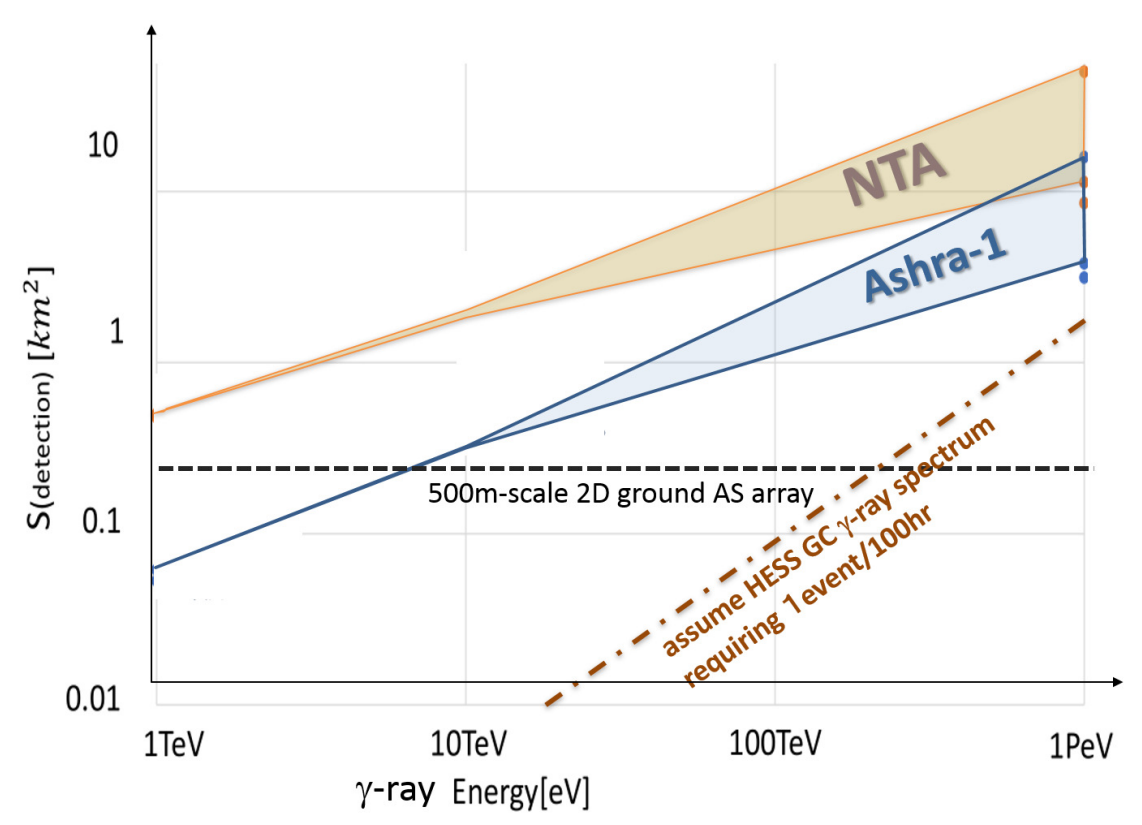

Figure 4: The $\gamma$-ray effective detection area versus energies comparing with NTA, Ashra-1 (bands), and 500m-scale 2D ground AS array (dash). Requirement of 1 events/100hr-observation assuming HESS GC $\gamma$-ray spectrum (dot-dash) [2] is also shown.

higher than $16 \mathrm{TeV}(240 \mathrm{TeV})$. This situation is useful to check the cut-off energy in the $\gamma$-ray spectrum in the Galactic bulge or central region as well as the large monitoring FOV and efficiency. Once the northward NTA units detect $v$ 's from the same source direction as $\gamma$-rays, we can discuss the physics of the occurrence of $\gamma$-rays and $v$ 's more concretely than ever.

\section{Dark Matter and New Particles}

The GB can also be considered an intriguing testing site for the discovery of elementary particles predicted by the non-standard theory of particle physics.

Weakly Interacting Massive Particles (WIMPs) are favoured DM candidates, which are preferentially discussed in the minimal supersymmetric standard model (MSSM) framework [24]. Indirect WIMP detection use secondary particles such as $\gamma \mathrm{s}, \nu \mathrm{s}$, weak bosons, tau pairs and so on from annihilations. Direct WIMP detection uses recoil nuclei from elastic WIMP-nucleus scattering. There is some complementarity between direct and indirect searches for DM, given the astrophysical assumptions inherent to the calculations. Both methods are sensitive to opposite extremes of the velocity distribution of DM particles in the Galaxy (low-velocity particles are captured more efficiently in the Sun, high-velocity particles leave clearer signals in direct detection experiments), as well as presenting different sensitivity to the structure of the DM halo (a local void or clump can deplete or enhance the possibilities for direct detection).

The announcement by the IceCube collaboration that they have detected more than 30 neutrino events of probable cosmic origin has been received with excitement, and has generated a great deal 
of interest in finding the source that caused them. Ordinary astrophysical (galactic or extragalactic) sources may be the cause of the signal (for review, [25]). However, the possibility of a DM origin is interesting because of its relevance to new physics beyond the Standard Model. The decay of PeV-class DM particles into neutrinos has recently been proposed [26, 27], while the case of PeV particles annihilating was rejected on the grounds of unitarity of the annihilation cross section. The simple assumption that DM is a thermal relic limits the maximum mass of the DM particle, which turns out to be a few hundred TeV for a thermal WIMP, the so called unitarity constraint. The claim was made while ignoring the contribution from the DM substructure, which in the cold DM scenario at $\mathrm{PeV}$ would result in lower free-streaming masses. Since the unitarity bound is not stringent at low velocities, these cold and dense subhaloes can therefore be the dominant contributors to the DM-induced neutrino flux, and can easily explain the events observed by IceCube [28]. On the other hand, DM particles might have never experienced local chemical equilibrium during the evolution of the Universe, and their mass may be in the range much larger than the mass of thermal WIMPs $[29,30]$. NTA can perform the sensitive indirect search for PeV-scale WIMP DM and non-standard new particles, with much better effective detection area for tau neutrinos from DM annihilation in the GB, especially above $10 \mathrm{PeV}$, the complementary sensitive energy region for IceCube.

Super-heavy particles $\left(\mathrm{M} \gtrsim 10^{4} \mathrm{GeV}\right)$ produced during inflation may be the dark matter, independent of their interaction strength. Most popular ones are strongly interacting super-heavy particles, magnetic monopoles, supersymmetric Q-balls and nuclearites.

Strongly interacting super-heavy particles will be captured by the Sun or condensed in the GB, and their annihilation in the center will produce a flux of energetic tau neutrinos that should be detectable by NTA [31].

Magnetic monopoles turn out to be consequence of most variants of Grand Unified Theories [32]. The electromagnetic energy losses of monopoles in the atmosphere, as well as neutrinos produced from monopole-antimonopole annihilations in the Sun and Earth, induce clear signatures in optical (Cherenkov and fluorescence) air-shower detectors like NTA [33].

Nuclearites (strange quark matter or strangelets) are hypothetical aggregates of $\mathrm{u}, \mathrm{d}$ and $\mathrm{s}$ quarks, combined with electrons to adjust electric neutrality. Nuclearites, like meteors, produce visible light as they traverse the atmosphere. Their luminosity as a function of their mass is $L=1.5 \times 10^{-3}(M / 1 \mu \mathrm{g})$ watt [34]. For example, the apparent visual magnitude of a $20 \mathrm{~g}$ nuclearite at a height of $10 \mathrm{~km}$ is -1.4 , equal to that of the brightest star, Sirius. Atmospheric nuclearites at galactic velocities $(v \sim 250 \mathrm{~km} / \mathrm{s}$ ) can easily be distinguished from ordinary meteors bounded to the Solar System, moving no faster than $72 \mathrm{~km} / \mathrm{s}$. It could be identified with clear evidence with the wide FOV high resolution optical detector of NTA.

Q-balls are hypothetical coherent states of quarks, sleptons and Higgs fields [35]. Neutral Q-ball (Supersymmetric Electrically Neutral Solitons, SENS) could catalyse proton decay along their path, similar to GUT monopoles. Electrically charged Q-ball (Supersymmetric Electrically Charged Solitons, SECS) would produce light in a similar way as nuclearites.

\section{Acknowledgement}

The Ashra Experiment was supported by the Coordination Fund for Promoting Science and Technology and by a Grant-in-Aid for Scientific Research and Grant-in-Aid for Scientific Research 
from the Ministry of Education, Culture, Sports, Science and Technology in Japan.

\section{References}

[1] M. Aartsen et al., PRL 113, 101101 (2014).

[2] HESS Collaboration, Nature 531, 476 (2016).

[3] IceCube Collaboration, Science 361, 147 (2018).

[4] M. Sasaki, ICRR2000 Sat. Sympo., 109 (2000).

[5] M. Sasaki, G. Hou, arXiv:1408.6244 (2014).

[6] J. Alvarez-Muniz et al. (GRAND Collaboration), arXiv:1810.09994.

[7] J. F. Krizmanic, UHECR 2018 (European Physical Journal Web of Conferences, Paris, 2018).

[8] C. R. Persichilli, Ph.D. thesis, University of California, 2018.

[9] P. Allison et al. (ARA Collaboration), Phys. Rev. D 93, 082003 (2016).

[10] R. A. Batista, R. M. de Almeida, B. Lago, and K. Kotera, J. Cosmol. Astropart. Phys. 01 (2019) 002.

[11] M. G. Aartsen et al. (IceCube Collaboration), Astrophys. J. 809, 98 (2015).

[12] M. Aartsen et al. (IceCube Collaboration), Phys. Rev. D 98, 062003 (2018).

[13] A. Aab et al. (Pierre Auger Collaboration), arXiv:1708.06592.

[14] P. W. Gorham et al. (ANITA Collaboration), arXiv:1902.04005.

[15] M. Sasaki, et al., Astropart. Phys. 19, 37 (2003).

[16] Y. Asaoka, M. Sasaki, Astropart. Phys. 41, 7 (2013).

[17] M. Sasaki, Prog. Theo. Phys. Suppl. 151, 192 (2003).

[18] Y. Asaoka, M. Sasaki, NIMA 647, 34 (2011).

[19] M. Sasaki, et al., NIMA 492, 49 (2002).

[20] M. Sasaki et al., NIMA 501, 359 (2003).

[21] A. M. Otte, PHYS. REV. D 99, 083012 (2019)

[22] Y. Aita et al., ApJ Lett. 736, L12 (2011).

[23] S. Ogawa et al., Pos (ICRC2019) 970 (2019).

[24] R.C. Cotta, J.S. Gainer, J.L. Hewett, and T.G. Rizzo. New Journal of Physics, 11(10):105026, 2009. 
[25] L. A. Anchordoqui et al., Journal of High Energy Astrophysics 1-2, 1 (2014).

[26] B. Feldstein, A. Kusenko, S. Matsumoto, and T.T. Yanagida, Phys. Rev. D 88, 015004 (2013).

[27] A. Esmaili and P. Dario Serpico, J. Cosmol. Astropart. Phys. 11 (2013) 054.

[28] J. Zavala, Physical Review D, 89(12):123516, 2014.

[29] D.J. Chung, E.W. Kolb, and A. Riotto. Physical Review D, 59(2):023501, 1998.

[30] P. Blasi, R. Dick, and E.W. Kolb. Astroparticle Physics, 18(1):57 - 66, 2002.

[31] I.F.M. Albuquerque, L. Hui, and E.W. Kolb. Phys. Rev. D, 64:083504, 2001.

[32] G. Giacomelli, S. Manzoor, E. Medinaceli, and L. Patrizii. Journal of Physics: Conference Series, 116(1):012005, 2008.

[33] S.D. Wick, T.W. Kephart, T.J. Weiler, and P.L. Biermann. Astroparticle Physics, 18(6):663 687, 2003.

[34] A. De Raújula and S.L. Glashow. Nature, 312(5996):734-737, 1984.

[35] A. Kusenko. Physics Letters B, 405(1-2):108 - 113, 1997. 